\title{
Long Interspersed Element-1 Methylation Level as a Prognostic Biomarker in Gastrointestinal Cancers
}

\author{
Yoshifumi Baba Taisuke Yagi Hiroshi Sawayama Yukiharu Hiyoshi \\ Takatsugu Ishimoto Masaaki Iwatsuki Yuji Miyamoto Naoya Yoshida \\ Hideo Baba
}

Department of Gastroenterological Surgery, Graduate School of Medical Sciences, Kumamoto University, Kumamoto, Japan

\section{Keywords}

Epigenetics · Methylation · Long interspersed element-1 . Gastrointestinal cancer · Prognosis

\section{Abstract}

Epigenetic changes play a crucial role in human cancer development. DNA methylation is a central epigenetic process that regulates levels of gene expression. Changes in DNA methylation that occur in human tumors include global DNA hypomethylation and site-specific CpG island promoter hypermethylation. Long interspersed element-1 (LINE-1) is a repetitive DNA retrotransposon that duplicates via a copyand-paste genetic mechanism. As LINE-1 constitutes approximately $17 \%$ of the human genome, the extent of LINE1 methylation is regarded as a surrogate marker of global DNA methylation. In a variety of gastrointestinal (Gl) cancers, LINE-1 hypomethylation is strongly associated with a poor prognosis, supporting its potential role as a prognostic biomarker. In this article, we summarize current knowledge regarding LINE-1 methylation and its prognostic impact in GI cancers.

(c) 2018 S. Karger AG, Basel

\section{Introduction}

Epigenetics is a promising and expanding research field in human cancer, and can be defined as the study of heritable changes in gene expression that do not involve changes to the underlying DNA sequence. One of the most widely studied epigenetic changes is DNA methylation, which is the postreplicative addition of a methyl group to the 5th carbon of the cytosine ring in CpG dinucleotides. CpG dinucleotides are widely and nonuniformly distributed throughout the human genome, and typically occur at a frequency of approximately one per 80 dinucleotides. However, isolated CpG-rich regions, referred to as "CpG islands," comprise $1-2 \%$ of the human genome. An estimated 45,000 $\mathrm{CpG}$ islands are located close to the promoter regions of various genes. DNA hypermethylation at promoter $\mathrm{CpG}$

This work was supported in part by a Grant-in-Aid for Scientific Research from the Japan Society for the Promotion of Science, grant number $17 \mathrm{H} 04273$.

\section{KARGER}

(c) 2018 S. Karger AG, Basel

E-Mail karger@karger.com

www.karger.com/dig
Yoshifumi Baba, MD, PhD, FACS

Department of Gastroenterological Surgery

Graduate School of Medical Sciences, Kumamoto University

1-1-1 Honjo, Kumamoto 860-8556 (Japan)

E-Mail y-baba@kumamoto-u.ac.jp 
sites suppresses the expression of tumor suppressor genes, thus contributing to cancer development and progression [1].

A second crucial DNA methylation change is genomewide DNA hypomethylation. Global DNA hypomethylation plays an important role in multistep tumorigenesis in a variety of ways. Accumulating evidence indicates that DNA hypomethylation of repetitive sequences (i.e., short interspersed transposable elements [SINEs or Alu elements] or long interspersed transposable elements [LINEs]) predisposes cells to chromosomal defects and rearrangements that lead to genetic instability [2]. Such increases in chromosomal instability contribute to cancer development and progression. Importantly, given that LINE-1 constitutes a substantial proportion (approximately 17\%) of the human genome [3], the degree of LINE-1 methylation is regarded as a surrogate marker of global DNA methylation.

In this review, we summarize the accumulated evidence for LINE-1 methylation level as a prognostic biomarker of gastrointestinal (GI) cancers.

\section{What Is LINE-1?}

Repetitive sequences constitute approximately half of the human genome and are subdivided into 2 principal types. One is the tandem repeat, or satellite, in which each repeat unit is immediately adjacent to another [4]. The other consists of interspersed repeats, which are repeated sequences that are spread throughout the genome [5]. Interspersed repeats are derived from transposable elements or mobile DNAs. The transposable elements comprise DNA transposons and retrotransposons, which are subdivided into sequences that contain long terminal repeats (LTR) and those that do not (non-LTR). The majority of mobile element activity in humans likely involves non-LTR retrotransposons, typified by LINE-1. LINE-1 is amplified in the human genome to more than 500,000 copies [6] and is present in a truncated form (mean length $=0.9 \mathrm{~kb}$ ) on both homologous chromosomes in the human genome. A limited proportion of LINE-1 exists in a potentially active form as full-length elements $(\sim 6 \mathrm{~kb})$. Thus, LINE-1 is regarded as a noninformative miscellany from the genetic past, and is often referred to as "junk" or "parasitic" DNA. However, accumulating evidence suggests an important role for LINE-1 in various cellular processes. LINE-1 can continuously rearrange the genome, thereby influencing the expression status of genes in different ways. LINE-1 may induce genetic vari- ation and polymorphism through the recombination and rearrangement as well as through endogenous mutagenesis [7]. In addition, LINE-1 expression can contribute to transcription disruption, insertion mutations, or DNA breaks, leading to genomic instability in cancer cells. The antisense promoter of LINE-1 can change gene expression status by modulating the transcriptional activity of surrounding genes [8].

\section{Methylation Status of LINE-1}

Full-length LINE-1 possesses an internal promoter in its 5 'UTR, which ranges from +1 to $909 \mathrm{bp}$. The initial 460 bp region of the 5'UTR includes 29 CpG sites, the methylation status of which has been extensively examined and shown to be high. Importantly, LINE-1 methylation levels in normal tissues strongly depend on tissue type: the range of LINE-1 methylation levels in certain tissues (e.g., esophagus, thyroid) is broad, while in other tissues (e.g., liver, kidney, breast, stomach, lung) it is limited [9]. In addition, LINE-1 methylation status has been proposed to differ at individual genomic locations: a study in human cancer cell lines has shown that methylation levels vary at 9 LINE-1 loci [10]. Another study evaluated the methylation patterns of 17 LINE-1 loci in several cell types and found that the methylation levels at these loci can be influenced differentially, depending on the location of the particular sequences in the genome [11]. Collectively, the variable methylation statuses of different sets of LINE-1 loci may give rise to different cellular phenotypes.

In 1993, Thayer et al. [12] demonstrated the methylation status of LINE-1 in cancer cells. Since then, tumoral LINE-1 hypomethylation has been reported in various human tumors, including those of the prostate, ovary, head and neck, lung, and testicle. Other research utilizing a broad range of cancer cell lines and clinical samples has demonstrated the wide variability of LINE-1 hypomethylation levels among normal and cancer tissue types [13].

\section{LINE-1 Methylation Level and Prognosis in GI Cancers}

Numerous studies have focused on the relationship between LINE-1 methylation level and clinical outcome in patients with human GI cancers (Table 1). In 2008, Ogino et al. [14] were the first to demonstrate that the extent of LINE-1 hypomethylation was linearly associat- 
Table 1. LINE-1 hypomethylation and patient prognosis in GI cancers

\begin{tabular}{lllll}
\hline Cancer type & $\begin{array}{l}\text { Number } \\
\text { of cases }\end{array}$ & Selection criterion & $\begin{array}{l}\text { Prognostic } \\
\text { impact of } \\
\text { hypomethylation }\end{array}$ & Reference \\
\hline Colon cancer & 643 & Stage III only & Unfavorable & {$[14]$} \\
Colon cancer & 161 & 343 & Unfavorable & {$[15]$} \\
Colorectal cancer & 207 & MSI cases only & Unfavorable & {$[16]$} \\
Colorectal cancer & 129 & Patients receiving adjuvant FOLFOX & Unfavorable & {$[17]$} \\
Colon cancer & 336 & Patients receiving adjuvant FOLFOX & Unfavorable & {$[19]$} \\
Colorectal cancer & 40 & Patients receiving adjuvant FOLFOX & Unfavorable & {$[20]$} \\
Colorectal cancer & 164 & Stage II only & Unfavorable & {$[18]$} \\
Colon cancer & 203 & Unfavorable & {$[22]$} \\
Gastric cancer & 198 & Advanced cases only & Unfavorable & {$[24]$} \\
Gastric cancer & 434 & Squamous cell carcinoma only & Unfavorable & {$[23]$} \\
Gastric cancer & 217 & Squavorable & {$[25]$} \\
Esophageal cancer & 105 & Squamous cell carcinoma only & Unfavorable & {$[26]$} \\
Esophageal cancer & 105 & & Unfavorable & {$[27]$} \\
HCC & 208 & & Unfavorable & {$[28]$} \\
HCC & 75 & & No impact & {$[29]$} \\
Pancreatic cancer & 126 & & & \\
\hline
\end{tabular}

HCC, hepatocellular carcinoma; MSI, microsatellite instability.

ed with aggressive tumor behavior, in an analysis of 2 independent prospective cohorts with 643 colon cancers. They observed an approximately fivefold difference in cancer-specific mortality, as LINE-1 methylation in the tumor genes ranged from high to low [14]. Subsequent studies have confirmed the relationship between LINE-1 hypomethylation and poor prognosis in resected colorectal cancers [15-18]. In addition, LINE-1 hypomethylation has been reported to correlate with unfavorable outcomes in colon cancer patients receiving oxaliplatin with fluorouracil and folinic acid adjuvant chemotherapy [1921].

Three studies have described the prognostic value of LINE-1 hypomethylation in patients with gastric cancer. Our group has reported that LINE-1 hypomethylation was associated with shorter survival, utilizing a database of more than 200 cases of gastric cancer, thus suggesting its potential for use as a prognostic biomarker [22]. This was in agreement with the findings of research groups in Korea [23, 24]. Similarly, regarding esophageal squamous cell carcinoma (ESCC), we showed that LINE-1 hypomethylation was significantly associated with lower disease-free survival and cancer-specific survival [25]. This finding was confirmed by Kawano et al. [26].

With respect to hepato-biliary-pancreatic cancers, Harada et al. [27] showed a significantly higher cancer recurrence rate in a LINE-1 hypomethylation group than in a hypermethylation group in a database of 208 hepatocellular carcinomas. Similar results in hepatocellular carcinoma have since been reported [28].

Only one study to date has focused on LINE-1 hypomethylation in pancreatic cancer. Interestingly, unlike in the other GI cancers, LINE-1 hypomethylation was not significantly associated with overall survival, cancer-specific survival, or disease-free survival in 126 patients with pancreatic cancer [29]. This discrepancy may reflect differences in tumor histology. The effect of LINE-1 methylation level on the survival of pancreatic cancer patients therefore needs to be confirmed in a larger cohort study.

\section{LINE-1 Hypomethylation and Aggressive Tumor Behavior}

The mechanism by which LINE-1 hypomethylation affects aggressive tumor behavior remains unclear. The link between LINE-1 hypomethylation and genomic instability represents one possible mechanism. Gaudet et al. [2] showed that transgenic mice carrying a hypomorphic DNA methyltransferase 1 displayed substantial genome-wide hypomethylation in all tissues. Interestingly, the mice developed aggressive T-cell lymphomas with a high frequency of chromosome 15 trisomy, thus supporting a causal role for DNA hypomethylation in 
tumor formation via the promotion of chromosomal instability [2]. In our previous study, we investigated LINE1-hypomethylated and LINE-1-hypermethylated ESCC tumors using array-based comparative genomic hybridization. LINE-1 hypomethylated tumors showed a high frequency of genomic gains at various loci containing candidate oncogenes such as CDK6. Taken together, these data suggest that global DNA hypomethylation in ESCC contributes to the acquisition of aggressive tumor behavior through the amplification of oncogenes such as CDK6 [30]. Another possible mechanism is transcriptional dysregulation, which might activate proto-oncogenes, endogenous retroviruses, or transposable elements that influence tumor aggressiveness. Given the epigenetic regulation of microRNA in human cancers, global DNA hypomethylation might contribute to the acquisition of aggressive tumor behavior via the aberrant expression of microRNA. Furthermore, in addition to acting as a surrogate marker for global DNA methylation, LINE-1 methylation status itself may exert biological effects. As noted above, LINE-1 elements are retrotransposons, which represent alternative promoters and contribute to non-coding RNA expression, thereby leading to the regulation of the function of multiple genes. Additional studies are needed to identify other potential mechanism(s) by which genome-wide DNA hypomethylation affects tumor behavior.

\section{Conclusions}

LINE-1 hypomethylation is associated with poorer prognosis in almost all types of GI cancer, supporting a role for LINE-1 methylation level as a prognostic biomarker. Importantly, epigenetic changes, unlike irreversible genetic changes, may represent reversible molecular targets for both cancer therapy and chemoprevention. Further investigations in this field should provide deeper insights into GI cancer development and contribute to the development of novel therapeutic strategies against these cancers.

\section{Disclosure Statement}

The authors have no conflicts of interest to disclose.

\section{References}

1 Rodriguez-Paredes M, Esteller M: Cancer epigenetics reaches mainstream oncology. Nat Med 2011;17:330-339.

2 Gaudet F, Hodgson JG, Eden A, JacksonGrusby L, Dausman J, Gray JW, et al: Induction of tumors in mice by genomic hypomethylation. Science 2003;300:489-492.

3 Cordaux R, Batzer MA: The impact of retrotransposons on human genome evolution. Nat Rev Genet 2009;10:691-703.

4 Gemayel R, Vinces MD, Legendre M, Verstrepen KJ: Variable tandem repeats accelerate evolution of coding and regulatory sequences. Annu Rev Genet 2010;44:445-477.

5 Singer MF: SINEs and LINEs: highly repeated short and long interspersed sequences in mammalian genomes. Cell 1982;28:433434.

6 Lander ES, Linton LM, Birren B, Nusbaum C, Zody MC, Baldwin J, et al: Initial sequencing and analysis of the human genome. Nature 2001;409:860-921.

7 Iskow RC, McCabe MT, Mills RE, Torene S, Pittard WS, Neuwald AF, et al: Natural mutagenesis of human genomes by endogenous retrotransposons. Cell 2010;141:12531261.

8 Weber B, Kimhi S, Howard G, Eden A, Lyko F: Demethylation of a LINE-1 antisense promoter in the cMet locus impairs Met signalling through induction of illegitimate transcription. Oncogene 2010;29:57755784.

9 Chalitchagorn K, Shuangshoti S, Hourpai N, Kongruttanachok N, Tangkijvanich $\mathrm{P}$, Thong-ngam D, et al: Distinctive pattern of LINE-1 methylation level in normal tissues and the association with carcinogenesis. Oncogene 2004;23:8841-8846.

10 Alves G, Tatro A, Fanning T: Differential methylation of human LINE-1 retrotransposons in malignant cells. Gene 1996;176:39-44.

11 Phokaew C, Kowudtitham S, Subbalekha K, Shuangshoti S, Mutirangura A: LINE-1 methylation patterns of different loci in normal and cancerous cells. Nucleic Acids Res 2008; 36:5704-5712.

12 Thayer RE, Singer MF, Fanning TG: Undermethylation of specific LINE-1 sequences in human cells producing a LINE-1-encoded protein. Gene 1993;133:273-277.

13 Baba Y, Murata A, Watanabe M, Baba H: Clinical implications of the LINE-1 methylation levels in patients with gastrointestinal cancer. Surg Today 2014;44:1807-1816.

14 Ogino S, Nosho K, Kirkner GJ, Kawasaki T, Chan AT, Schernhammer ES, et al: A cohort study of tumoral LINE-1 hypomethylation and prognosis in colon cancer. J Natl Cancer Inst 2008; 100:1734-1738.

15 Ahn JB, Chung WB, Maeda O, Shin SJ, Kim HS, Chung HC, et al: DNA methylation pre- dicts recurrence from resected stage III proximal colon cancer. Cancer 2011;117:18471854.

16 Antelo M, Balaguer F, Shia J, Shen Y, Hur K, Moreira L, et al: A high degree of LINE-1 hypomethylation is a unique feature of early-onset colorectal cancer. PLoS One 2012;7: e45357.

17 Rhee YY, Kim MJ, Bae JM, Koh JM, Cho NY, Juhnn YS, et al: Clinical outcomes of patients with microsatellite-unstable colorectal carcinomas depend on L1 methylation level. Ann Surg Oncol 2012;19:3441-3448.

18 Swets M, Zaalberg A, Boot A, van Wezel T, Frouws MA, Bastiaannet E, et al: Tumor LINE-1 methylation level in association with survival of patients with stage II colon cancer. Int J Mol Sci 2016;18.

19 Lou YT, Chen CW, Fan YC, Chang WC, Lu CY, Wu IC, et al: LINE-1 methylation status correlates significantly to post-therapeutic recurrence in stage III colon cancer patients receiving FOLFOX-4 adjuvant chemotherapy. PLoS One 2014; 10:e0123973.

20 Chen D, Wen X, Song YS, Rhee YY, Lee $\mathrm{TH}$, Cho NY, et al: Associations and prognostic implications of Eastern Cooperative Oncology Group performance status and tumoral LINE-1 methylation status in stage III colon cancer patients. Clin Epigenetics 2016;8:36. 
21 Kaneko M, Kotake M, Bando H, Yamada T, Takemura H, Minamoto T: Prognostic and predictive significance of long interspersed nucleotide element-1 methylation in advanced-stage colorectal cancer. BMC Cancer 2016; $16: 945$.

22 Shigaki H, Baba Y, Watanabe M, Murata A, Iwagami S, Miyake K, et al: LINE-1 hypomethylation in gastric cancer, detected by bisulfite pyrosequencing, is associated with poor prognosis. Gastric Cancer 2013;16:480487.

23 Song YS, Kim Y, Cho NY, Yang HK, Kim WH, Kang GH: Methylation status of long interspersed element- 1 in advanced gastric cancer and its prognostic implication. Gastric Cancer 2016;19:98-106.

24 Bae JM, Shin SH, Kwon HJ, Park SY, Kook MC, Kim YW, et al: ALU and LINE-1 hypo- methylations in multistep gastric carcinogenesis and their prognostic implications. Int J Cancer 2012;131:1323-1331.

25 Iwagami S, Baba Y, Watanabe M, Shigaki $H$, Miyake K, Ishimoto T, et al: LINE-1 hypomethylation is associated with a poor prognosis among patients with curatively resected esophageal squamous cell carcinoma. Ann Surg 2012, in press.

26 Kawano H, Saeki H, Kitao H, Tsuda Y, Otsu $\mathrm{H}$, Ando K, et al: Chromosomal instability associated with global DNA hypomethylation is associated with the initiation and progression of esophageal squamous cell carcinoma. Ann Surg Oncol 2014;21(suppl 4):S696S702.

27 Harada K, Baba Y, Ishimoto T, Chikamoto A, Kosumi K, Hayashi H, et al: LINE-1 methylation level and patient prognosis in a database of 208 hepatocellular carcinomas. Ann Surg Oncol 2015;22:1280-1287.

28 Zhu C, Utsunomiya T, Ikemoto T, Yamada S, Morine Y, Imura S, et al: Hypomethylation of long interspersed nuclear element-1 (LINE-1) is associated with poor prognosis via activation of c-MET in hepatocellular carcinoma. Ann Surg Oncol 2014;21(suppl 4):S729-S735.

29 Yamamura K, Kosumi K, Baba Y, et al: LINE1 methylation level and prognosis in pancreas cancer: pyrosequencing technology and literature review. Surg Today 2017, Epub ahead of print.

30 Baba Y, Watanabe M, Murata A, Shigaki H, Miyake K, Ishimoto T, et al: LINE-1 hypomethylation, DNA copy number alterations, and CDK6 amplification in esophageal squamous cell carcinoma. Clin Cancer Res 2014; 20:1114-1124 